\title{
Targeting Cancer with the Resources at Hand: Rolando Camacho, MD Chair, National Oncology Group
}

\author{
By Gail Reed, MS
}

Rolando Camacho was born in Pinar del Río-Cuba's westernmost province and, ironically enough, home to the country's richest tobacco-producing farmlands. He was about to leave for an engineering scholarship abroad when he accompanied a friend to medical school on the first day of classes in Havana, a bus ride that changed his life. Since completing his oncology residency in 1985, Dr Camacho has served as director of Pinar del Río's Cancer Control Program, chair of the National Cancer Control Program, director of the National Oncology and Radiobiology Institute (INOR), and today, chair of the National Oncology Group. The Group advises the Ministry of Public Health through its National Cancer Control Unit on questions of cancer control policy, planning and development of human resources for cancer care, structure of services, material resource planning and cancer research. Since 1988, breast cancer has been a particular focus for Dr Camacho's research, practice and teaching.

MEDICC Review spoke with Dr. Camacho for his views on the problem of cancer in Cuba, and the prospects for more effective control.

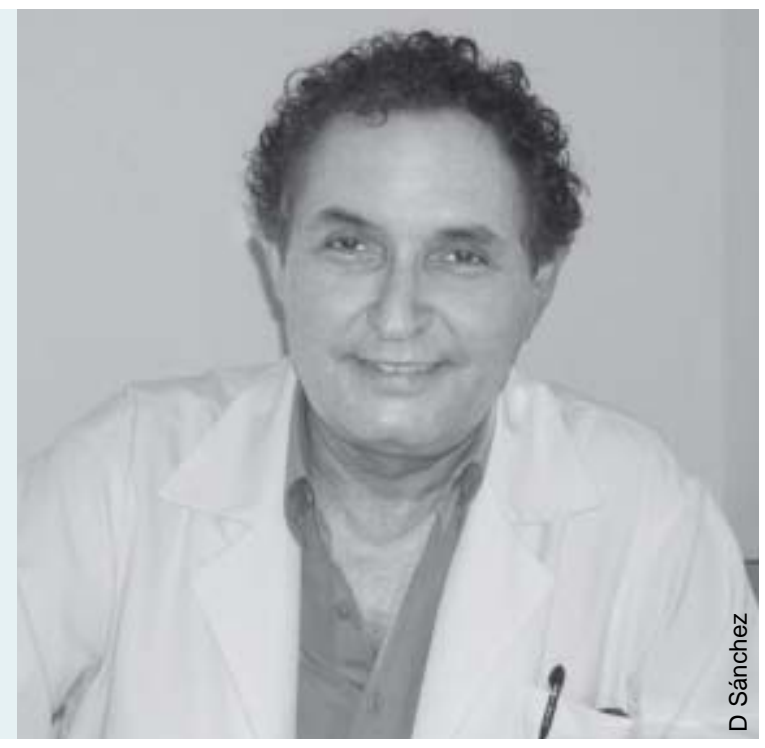

\section{MEDICC Review: How would you sum up the problem of can- cer in Cuba today?}

Rolando Camacho: Despite our efforts over the decades, we are not where we would like to be. Today, one of every four deaths in Cuba is from cancer; one of every three deaths in the 45-64 year age bracket.

When we examine trends over time for the main causes of death-heart disease, malignant tumors and cerebrovascular disease, in that order-we see that now, cancer has essentially caught up with heart disease as the main killer. Or put differently: while we have been able to reduce mortality from the other two conditions, we have not been successful in significantly reducing cancer mortality. Furthermore, of the main causes of death, cancer also has the highest-and increasing—rate of years of potential life lost (YPLL).

These trends can be explained in part by the economic crisis we suffered in the 1990s, which seriously affected screening programs, pharmaceutical production and imports, and also availability and repair of medical equipment. Only in the last decade have we been able to gradually increase budgets for radiotherapy and cancer medications. For example, in the year 2000, we had only 36 products available for cancer treatment; by 2007 , we had 57 , enough to cover the standard treatments for all cancer patients. Stepped-up national production of chemotherapy agents and biotechnology products has contributed to this result. We're now focusing more efforts on improving infrastructure for cancer surgery and nuclear medicine throughout the country.

But this is not enough, of course. The most important thing is not to treat patients but rather not have patients to treat, or at least to diagnose them earlier.
MEDICC Review: Which leads us to strategies for better prevention and earlier diagnosis. What needs to be done to reduce cancer and cancer mortality in Cuba, and by extension, in other developing countries?

Rolando Camacho: This is a particularly sensitive question, since Cuba is a developing country in the midst of a worldwide economic and financial crisis. And the World Health Organization indicates that $70 \%$ of new cancer cases and $75 \%$ of cancer deaths over the next 25 years will come from developing nations. So the challenge is a familiar one: develop effective strategies with a fraction of the resources at the disposal of richer countries.

Yes, product " $X$ " may prolong survival in the Global North, but how did it work elsewhere? We know that broad mammography screening can reduce mortality. But where? In what setting? That's not a model that can be used in poor countries. Neither the strategies nor the results are transferable to our context.

And this is true for every cancer in every developing country: we need to forge a different mentality that depends on the resources we DO have, to create a culture of healthy living and prevention. We need to document what works and accumulate a scientific evidence base to guide us along our own path, since the strategies in place in wealthier nations simply can't be expected to work for us.

That said, I think Cuba has some advantages, which should make it possible for us to play a more significant role in the search for viable approaches and models. For a start, we have a well-educated population. We also have the human resources needed. And our public health system provides free, universal coverage at all levels. 
Smoking in Cuba: Reduction Goals for 2015

\begin{tabular}{|c|c|c|}
\hline \multirow[t]{2}{*}{ Baseline } & \multicolumn{2}{|c|}{ Goals } \\
\hline & For 2010 & For 2015 \\
\hline $32 \%$ & $20 \%$ & $40 \%$ \\
\hline 32 & 25.6 & 19.2 \\
\hline
\end{tabular}

Indicator: Prevalence of smokers in Cuban population aged $\geq 17$ years

Source: 2nd National Survey on Risk Factors and Chronic Diseases, 2001

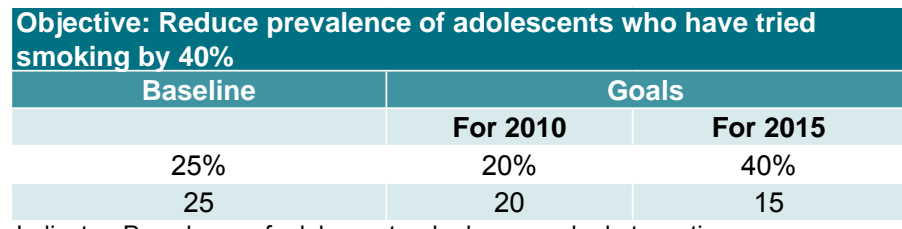

Indicator: Prevalence of adolescents who have smoked at any time

Source: The Global Youth Tobacco Survey, Cuba, 2004

$\begin{aligned} & \text { Objective: Reduce prevalence of smokers among health } \\
& \text { professionals by } 60 \%\end{aligned}$
\begin{tabular}{c|cc|}
\hline Baseline & For 2010 & Goals \\
\hline $31 \%$ & $20 \%$ & $60 \%$ \\
31 & 24.8 & 12.4
\end{tabular}

Indicator: Prevalence of smokers among health professionals

Source: Provincial Survey on Smoking among Physicians and Nurses, City of Havana, 2005

\begin{tabular}{|c|cc|}
\hline \multicolumn{3}{|c|}{ Objective: Reduce prevalence of passive smoking by $\mathbf{1 2} \%$} \\
\hline & \multicolumn{3}{c|}{ Goals } \\
\hline Baseline & For $\mathbf{2 0 1 0}$ & For $\mathbf{2 0 1 5}$ \\
$88 \%$ & $7 \%$ & $12 \%$ \\
88 & 81.8 & 77.4 \\
\hline
\end{tabular}

Indicator: Prevalence of persons exposed to tobacco smoke in the environment. Source: The Global Youth Tobacco Survey, Cuba, 2004.

Objective: Reduce percentage of persons who start smoking in the 20 to 24 -year age group to $20 \%$

\begin{tabular}{|c|cc|}
\hline & \multicolumn{3}{c|}{ Goals } \\
\hline Baseline & For 2010 & For 2015 \\
$22.2 \%$ & $21 \%$ & $20 \%$ \\
\hline
\end{tabular}

Indicator: Percent of persons aged 20-24 years who start smoking

Source: 2nd National Survey on Risk Factors and Chronic Diseases, 2001

Translated from the report Proyecciones de la salud pública en Cuba para el 2015

(Public Health Projections in Cuba for Year 2015)

MEDICC Review: The WHO says that $42 \%$ of cancers are preventable and a good share are curable. Where to start?

Rolando Camacho: We need to start with the facts. Here in Cuba, that means detailed data from the National Cancer Registry. Everything we assess-needs for equipment, human resources, medicines-starts with Registry statistics showing cancer trends in Cuba.

Then our job is to zero in on the cancers doing the most damage: the sites by sex and age group, and geographic stratification of risk for each type of cancer. The prevalence of cancer-associated risk factors is a key element, of coursewhether smoking, environmental exposure to carcinogens, sexual conduct, obesity and other dietary issues, or lack of exercise. General demographic trends are also considered: in this case, the rapid aging of the Cuban population. Then we have to take a very close look at mortality rates, which provide clues to effectiveness of early diagnosis strategies and treatments.

Based on analysis of all these factors, the National Cancer Control Unit is generating recommendations for more effective public health prevention and care strategies. The actions needed may not be all that many, but they have to be coordinated, and they have to focus on the most important things.

For example, reviewing data from 2007, you can see that geographically, over $86 \%$ of high mortality risk from cancer is concentrated in 25 of Cuba's 169 municipalities, and that over $80 \%$ is in Havana City municipalities. What's more, over half of all cancer mortality in Cuba is found in the top five cancer sites: lung and colorectal cancers in the whole population, plus prostate in men, and breast and cervical cancers in women. Taking a closer look: the number one cancer killer in Cuba is lung cancer, and its association with high smoking prevalence is well established.

MEDICC Review: Let's take a deeper look at lung cancer and its causes. How effective have no-smoking campaigns been?

Rolando Camacho: Well, we have reduced smoking among people aged 17 years and older from over $66 \%$ in the 1970 s to $32 \%$ in 2001 , but that rate is still very high. Health workers smoke. Even some leaders smoke. In 2008 , more than 5,000 people died from lung cancer, with the highest mortality in people under 70 years of age. This also has a serious impact on years of potential life lost, and on the country's economy.

In the last decade observed, mortality increased in men less than $1 \%$, but increased in women by $2.3 \%$. We estimate that if smoking prevalence were reduced to $19 \%$ by 2015 -our goal-then we would be saving 1,600 lives annually. In fact, in the Public Health Projections for Cuba for Year 2015, it states: "An effective anti-smoking policy is the health intervention with the greatest impact on population health."

Again, the devil is in the details. The National Cancer Control Unit is working to coordinate all the necessary players to make the no-smoking effort more successful. Before, our efforts were more fragmented.

MEDICC Review: What about breast cancer, which has the highest cancer incidence and second highest mortality among Cuban women?

Rolando Camacho: Nearly $70 \%$ are diagnosed in earlier stages (0-2), which means we are doing better than Latin America as a whole and than other developing countries. However, mortality has slightly increased since 2004-from 14.1 to 14.9 per 100,000 population in 2008 (rates adjusted to world population). We are also seeing an increase in incidence in women over 50 , and an increase in diagnosis at advanced stages in the same age group.

To reverse course, we need to increase the practice of selfexamination and clinical examination by family doctors at the primary care level-to at least $80 \%$ of women 30 years and older; and we need to purchase and install more mammography units across the country to guarantee confirmation of diagnosis and use for more selective screening. 


\section{MEDICC Review: And the other main cancer sites?}

Rolando Camacho: In terms of cervical cancer in women, our incidence and mortality are among the lowest in the Americas, right above the USA and Canada. We are treating many lesions in the pre-cancerous stages, and $70 \%$ are detected in stages $0-1$. This is the reverse of other developing countries. Yet, the national cervical cancer screening program, based at the primary care level, now covers only about $70 \%$ of women in the $25-59$ age group. To be effective in reducing mortality, this needs to reach $80 \%$ coverage. In highly developed countries, cervical cancer mortality was reduced by reaching this kind of coverage in screening programs. The new HPV vaccine is not an option for Cuba at this point-or for broad use in any developing country-since its import cost is prohibitive. We also have not seen the results of the vaccine over time.

Prostate cancer is the second cause of cancer mortality in men, and the largest share of deaths is in men over 70 years old. Most prostate cancer progresses slowly, so survival rates can be increased by greater public education, convincing men to be examined as soon as any symptoms appear or even when they are asymptomatic. A Cuban prostate-specific antigen (PSA) test is now available and being used.

Colorectal cancer-the third cause of cancer deaths in both men and women-is essentially related to dietary habits. You can see this in the United States, for example, where frequency of this cancer is diminishing among the more affluent, but rising among poorer people. Reducing colorectal cancer and associated mortality depend on education to change habits; availability of more fruits and vegetables; and more education for health professionals in terms of evaluating symptoms. Even without moving to mass screening-which is very costly-we can gain significant ground through education.

\section{MEDICC Review: How does Cuba pursue international coop- eration in cancer care and research?}

Rolando Camacho: We have significant cooperation in the field with Costa Rica, El Salvador, the Dominican Republic, Angola and other developing countries. We are working with Mexico in basic immunology and palliative care, and with Brazil in cancer control strategies and tumor banks. Collaboration extends to Canada (in genetics research), France (cancer epidemiology, among others), Switzerland (clinical oncology), and organizations such as the WHO and its International Agency for Research in Cancer, the Latin American Alliance against Cancer, and the International Atomic Energy Agency (in radiotherapy and nuclear medicine).

MEDICC Review: In its advisory capacity, one of the main areas for the National Oncology Group is development of oncology and related medical fields. How are oncologists trained in Cuba, and are there changes in the offing?

Rolando Camacho: The residency program is currently four years, in which residents receive general oncology training and then specialize in surgery, clinical oncology or radiotherapy. We are now in the process of changing this to a three-year program with these three concentrations and a fourth year added to the surgery residency. This is more in tune with oncology residency programs internationally requiring greater specialization, although of course, the common thread of general oncology will be maintained in each.

MEDICC Review: Summing up, if you were to recommend three main things to reduce cancer mortality in Cuba, what would these be?

Rolando Camacho: First, reduce smoking. Second, achieve effective public education about cancer. And third, attain quality coverage of cervical and breast cancer screening programs, reaching $80 \%$ of target populations for Pap tests, breast self-exams and primary-care clinical breast exams. -1 - 